\title{
Modification of Emission Properties of ZnO Layers due to Plasmonic Near-Field Coupling to Ag Nanoislands
}

\author{
Joanna Papierska • Bartłomiej S. Witkowski • \\ Anastasiya Derkachova • Krzysztof P. Korona • \\ Johannes Binder • Krzysztof Gałkowski • \\ Lukasz Wachnicki • Marek Godlewski • Tomasz Dietl • \\ Jan Suffczyński
}

Received: 26 November 2012 / Accepted: 30 January 2013 /Published online: 12 February 2013

(C) The Author(s) 2013. This article is published with open access at Springerlink.com

\begin{abstract}
A simple fabrication method of silver (Ag) nanoislands on $\mathrm{ZnO}$ films is presented. Continuous wave and time-resolved photoluminescence and transmission are employed to investigate modifications of visible and UV emissions of $\mathrm{ZnO}$ brought about by coupling to localized surface plasmons residing on Ag nanoislands. The size of the nanoislands, determining their absorption and scattering efficiencies, is found to be an important factor governing plasmonic modification of optical response of $\mathrm{ZnO}$ films. The presence of the Ag nanoislands of appropriate dimensions causes a strong (threefold) increase in emission intensity and up to 1.5 times faster recombination. The experimental results are successfully described by model calculations within the Mie theory.
\end{abstract}

J. Papierska $\cdot$ K. P. Korona · J. Binder · K. Gałkowski •

J. Suffczyński $(\square)$

Institute of Experimental Physics, Faculty of Physics,

University of Warsaw, Hoża 69 St.,

00-681 Warszawa, Poland

e-mail: Jan.Suffczynski@fuw.edu.pl

T. Dietl

Institute of Theoretical Physics, Faculty of Physics,

University of Warsaw, Hoża 69 St.,

00-681 Warszawa, Poland

B. S. Witkowski • A. Derkachova $\cdot$ Ł. Wachnicki ·

M. Godlewski · T. Dietl

Institute of Physics, Polish Academy of Sciences,

Lotników 32/46 Av.,

02-668 Warszawa, Poland
Keywords Zinc oxide - Silver nanoislands · Photoluminescence $\cdot$ Mie theory $\cdot$ Exciton

\section{Introduction}

Recent intensive research in the domain of plasmonics has shown that optical effects in semiconductors are strongly modified by coupling of light to plasma oscillations in metallic nanostructures residing on or in the proximity of the semiconductor surface $[1,2]$. In particular, a considerable enhancement of the emission intensity in the case of either self-organized [3] or lithographically defined metal nanostructures [1] was observed. Many factors such as density, shape, size and its distribution, agglomeration, internal structure, as well as composition and porosity of the surface of metal nanostructures determine their impact on the optical response of semiconductors $[1,4]$. A number of publications on $\mathrm{ZnO}$-metal hybrid structures devoted to different aspects of the relevant physics have appeared within the last decade. In particular, continuous wave photoluminescence (PL) studies of silver $(\mathrm{Ag}) / \mathrm{ZnO}$ demonstrated an enhancement of subband gap emission by $\mathrm{Ag}$ nanoislands $[4,5]$. It was speculated that this enhancement is due the resonant coupling of spontaneous emissions in $\mathrm{ZnO}$ to the surface plasmons in metal overlayers $[4,5]$.

Here, we apply the known [4] and an innovative method for fabrication on $\mathrm{ZnO}$ thin films of $\mathrm{Ag}$ islands with diameter of 10-60 and 60-120 nm, respectively. By employing PL, time-resolved PL, and transmission measurements, we determine experimentally the impact of the Ag coating on the $\mathrm{ZnO}$ film optical response in the visible and ultraviolet 
range. We describe the set of our experimental data within the Mie theory. The employed model shows that the size and resulting efficiencies of the light scattering and absorption by metal nanoparticles are decisive for modifying optical performance of semiconductors.

\section{Samples}

The studied $\mathrm{ZnO}$ layers are grown by atomic layer deposition on a 3- $\mu \mathrm{m}$ galium nitride $(\mathrm{GaN})$ template [6]. The $\mathrm{ZnO}$ layers of $50 \mathrm{~nm}$ thickness are studied in order to assure that the whole layer experiences the plasmonic mode decaying within the range of tens of nanometers from the sample surface [7].

Two methods have been employed to cover samples by $\mathrm{Ag}$ nanoislands. Sample A is covered with silver islands by sputter deposition lasting for $2 \mathrm{~s}$. Sample B is prepared in a more elaborate way, exploring an original method of metal island formation. The film surface is first coated by $\mathrm{Ag}$ sputtering over 15-20 s, yielding a 3-nm layer of $\mathrm{Ag}$, and it is then annealed at a temperature of $750{ }^{\circ} \mathrm{C}$ for $3 \mathrm{~min}$. The annealing process results in the formation of metal islands. Actually, in the case of both samples, half of the film is masked in a way preventing $\mathrm{Ag}$ deposition. This part of the $\mathrm{ZnO}$ surface, uncovered by $\mathrm{Ag}$ nanoislands, serves as a reference. As determined by scanning electron microscope (SEM) measurements (Fig. 1), islands of diameter distributed between $D=10$ and $60 \mathrm{~nm}$ (with dominating $D=10 \mathrm{~nm}$ ) with a surface density of $\rho \approx 2,000 / \mu \mathrm{m}^{2}$ are obtained by the first method (sample A). In the case of the second method, islands of diameter distributed equally between 60 and $120 \mathrm{~nm}$ with a surface density of $\rho \approx 25 / \mu \mathrm{m}^{2}$ are obtained (sample B). The Ag islands have a quasi-spherical shape in both samples.

The presence of Ag nanoislands on the surfaces of both samples is confirmed by energy-dispersive X-ray (EDX) measurements conducted with a beam of energy of $5 \mathrm{keV}$. A comparison of the EDX profiles (see Fig. 2) measured on
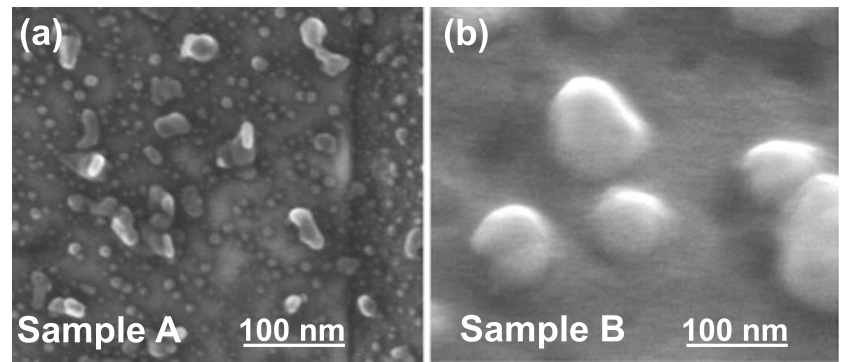

Fig. 1 SEM image of sample A (a) and sample B (b). The magnitudes of diameter and surface density of $\mathrm{Ag}$ nanoislands are determined to be $10-60 \mathrm{~nm}$ and $2,000 / \mu^{2}$ (sample $A$ ) or $60-120 \mathrm{~nm}$ and $25 / \mu \mathrm{m}^{2}$ (sample B), respectively

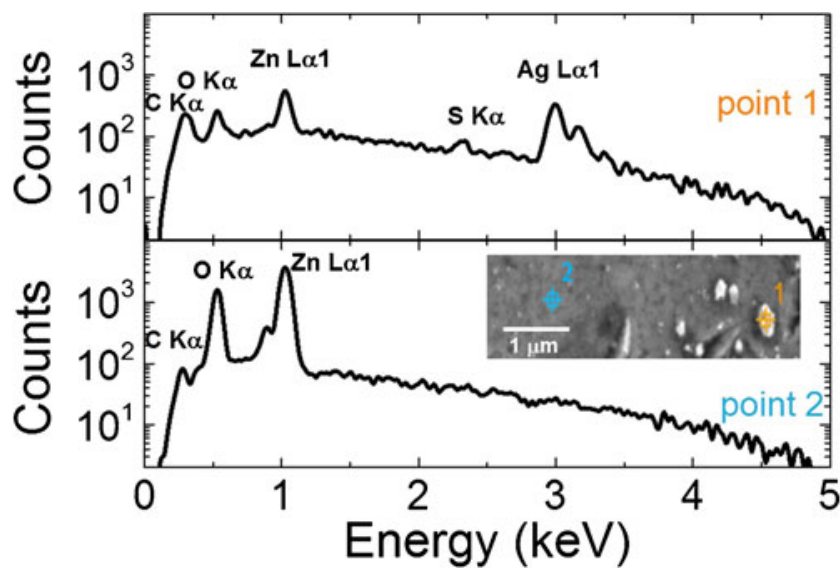

Fig. 2 EDX spectra measured at two points on sample B: with (point 1) and without (point 2) Ag nanoisland. The inset: SEM image of the sample region with indicated points 1 and 2

the representative points 1 and 2 on the sample B (see inset to Fig. 2) shows that a clear maximum at around $3 \mathrm{keV}$ related to $\mathrm{Ag}$ is evidenced only in the case of a sample region covered with the nanoisland. The $\mathrm{Ag}$ maximum is assisted by a weak satellite maximum at around $2.3 \mathrm{keV}$ related to sulfide, what is presumably due to a passivation of nanoisland surface by sulfite atoms from the air. Due to the strong background signal related to oxygen from $\mathrm{ZnO}$, it is impossible to determine the degree of oxidation of the $\mathrm{Ag}$ nanoislands.

\section{Experiment}

Photoluminescence, time-resolved PL, and transmission spectra are measured at temperatures ranging from 4 to $300 \mathrm{~K}$. The emission is excited at $325 \mathrm{~nm}$ (He-Cd laser) or at $300 \mathrm{~nm}$ (frequency-tripled Ti:sapphire-pulsed laser) in the case of PL and time-resolved PL, respectively. A halogen lamp serves as the light source in the transmission experiment. The incident beam is focused on the sample surface to a spot of $0.5 \mathrm{~mm}$ (in the case of time-resolved PL $3 \mu \mathrm{m}$ ) diameter. The signal arising from the sample is dispersed by a grating monochromator (600 or 1800 grooves $/ \mathrm{mm}$ ) and recorded by a CCD camera or a streak camera in the case of time-resolved PL.

\section{Results}

In order to characterize optical properties of the hybrid structures, the transmission in the energy range $1.5-3.6 \mathrm{eV}$ has been measured. We estimate that for the thickness and the absorption coefficient [8] of the $\mathrm{ZnO}$ layer, its absorption is negligible. Accordingly, for energies below the band gap of the GaN template $(\sim 3.42 \mathrm{eV}$ at $300 \mathrm{~K})$, the observed extinction results from the interaction of light with the $\mathrm{Ag}$ nanoislands. The ratio of the transmission of the sample B 
part covered with Ag nanoislands to the transmission of the uncovered part exhibits a wide $(0.6 \mathrm{eV})$ minimum (modulation $60 \%$ ) at around $\sim 2.3 \mathrm{eV}$, indicating the position of the extinction maximum. In the case of sample A, the extinction related to the Ag nanoislands is much weaker and shows no clear minimum.

Continuous wave PL spectra taken for both studied samples at $5 \mathrm{~K}$ are presented in Fig. 3a, c. The spectra collected for Ag-covered and uncovered regions are shown by solid and dotted lines, respectively. The dominating transition at $\sim 3.36 \mathrm{eV}$ is attributed to bound exciton emission in $\mathrm{ZnO}$. The signal observed in the $\sim 2.90-3.33 \mathrm{eV}$ energy range is assigned to stacking faults. A weak defect-related signal appears below $\sim 2.9 \mathrm{eV}$. A more detailed analysis of the near-band gap emission is presented below. As expected and seen in Fig. 3a, c, excitonic emission shows a shift towards lower energies and broadening when temperature is increased to $300 \mathrm{~K}$.

In order to determine the impact of $\mathrm{Ag}$ nanoparticles on the emission from $\mathrm{ZnO}$ films, the ratio of the photoluminescence from the covered to uncovered part of the sample is evaluated for both samples (Fig. 3b, d). As seen in Fig. 3b, the presence of 10-60 nm Ag islands affects the PL signal of sample A only weakly, leading to its slight decrease in the almost entire registered spectral range. Much more pronounced effects are observed in the case of sample B, covered with $60-120 \mathrm{~nm} \mathrm{Ag}$ islands. As seen in Fig. 3d, the presence of $\mathrm{Ag}$ nanoparticles exhibits up to threefold emission enhancement in the spectral range below $\sim 2.4 \mathrm{eV}$. However, in the spectral range above $\sim 2.4 \mathrm{eV}$ including the excitonic region, a quenching of the emission is observed. A comparison of the results for 2 and $300 \mathrm{~K}$ shown in Fig. 3 indicates that the observed effects of the PL enhancement or quenching are basically temperature independent. This is understood, provided that their origin is related to plasmonic
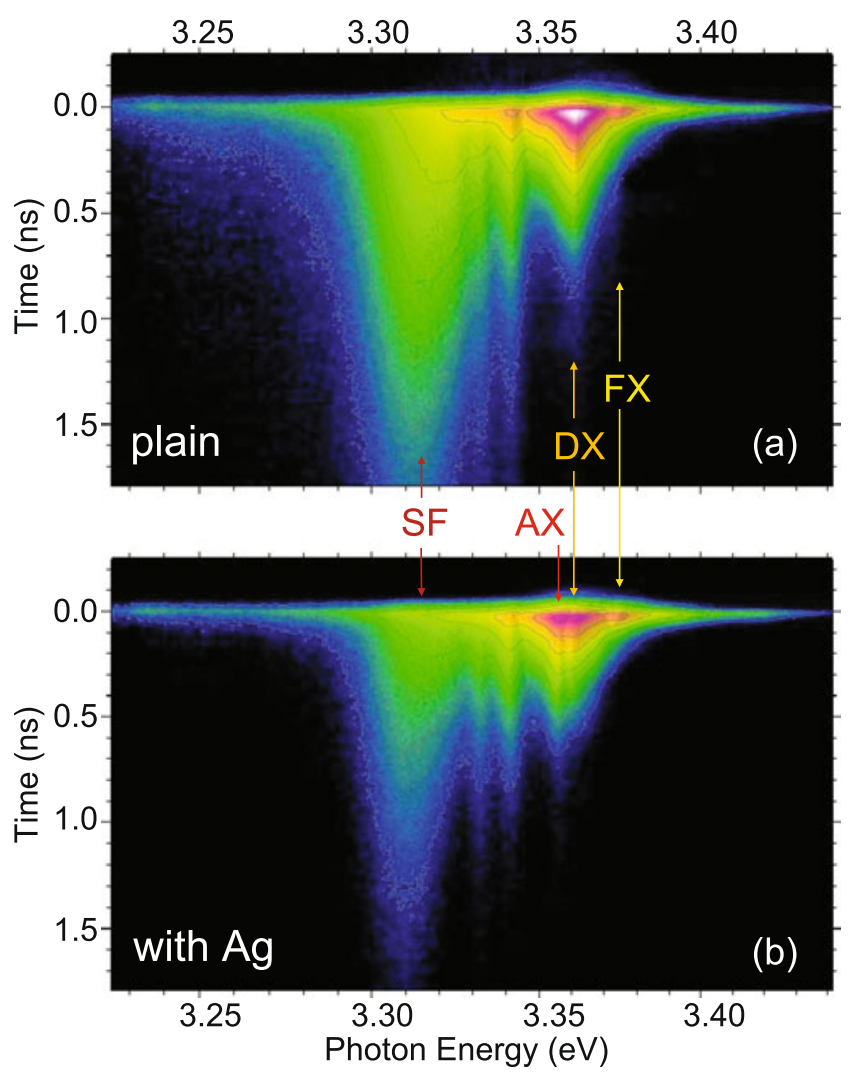

Fig. 4 Time-resolved PL spectra taken for sample B at $4 \mathrm{~K}$ for regions: plain (a) and covered (b) by Ag nanoislands of diameter $D=60$ $120 \mathrm{~nm}$. The contour values are set by an exponential rule, and the ratio between the two nearest contour curves is $r=10^{1 / 2}$

effects in Ag, which are governed by the magnitude of electron density, independent of temperature. A model presented in "Theoretical model" section corroborates the plasmonic origin of the above observations.

Results of time-resolved PL measurements at $4 \mathrm{~K}$ shed more light on the nature of the signal quenching in the near-
Fig. 3 Photoluminescence spectra of the $\mathrm{ZnO}$ layers: sample A (a) and sample B (c) as grown (solid line) and covered with Ag nanoparticles (dashed line). Ratio of the photoluminescence intensity from the covered to uncovered parts of sample A (b) and B (d). The spectra registered at $T=5$ and $300 \mathrm{~K}$ are indicated in black and red (or gray), respectively
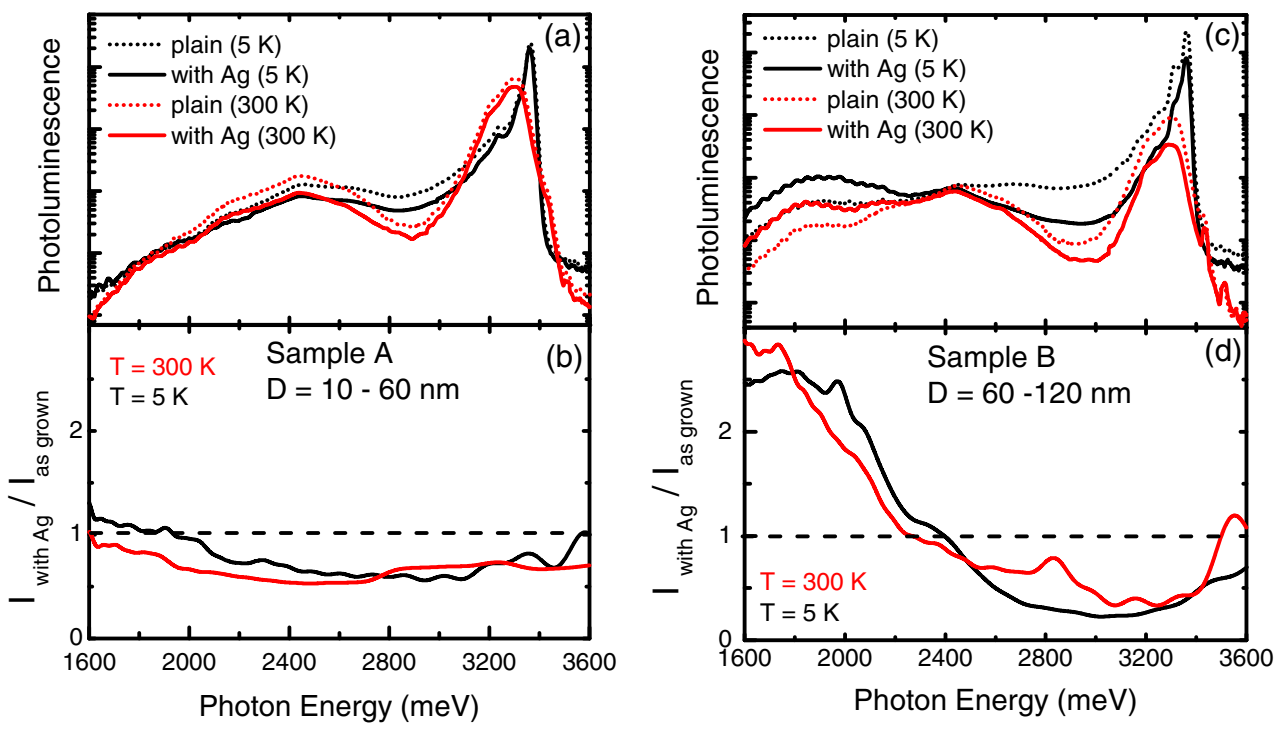
band gap spectral region resulting from the presence of the Ag nanoparticles. In Fig. 4, a temporal evolution of the PL spectra following the excitation pulse is shown for both covered and uncovered parts of sample B. A few dominant peaks are present in the signal coming from both parts of the sample. As indicated in Fig. 4, free exciton (FX) and donor bound exciton (DX) transitions are observed at 3.375 and $3.361 \mathrm{eV}$, respectively [9]. Two weak peaks are observed at 3.33 and $3.34 \mathrm{eV}$; their origin is not clear. Stacking fault (SF)-related emission is observed in the spectral range 3.30-3.33 eV [10]. Furthermore, acceptor bound exciton (AX) [9], not observed in the signal from the uncovered sample, appears at $3.356 \mathrm{eV}$ in the emission from the Ag-covered sample. This results most probably from the diffusion of the Ag atoms, acting as acceptor centers, to the $\mathrm{ZnO}$ layer during the process of the sample annealing.

The analysis of time-resolved PL for sample B (see Fig. 4) reveals that the decay of free excitons is monoexponential and relatively fast $(25 \pm 5$ and $35 \pm 5 \mathrm{ps}$, for the Agcovered and uncovered region, respectively). The DX decays are composed of two components. The shorter decay constant $\tau_{1}$ is $66 \pm 5$ and $77 \pm 5$ ps for the Ag-covered and uncovered regions, respectively. The longer component has the lifetime $\tau_{2}$ of about $0.64 \pm 0.05 \mathrm{~ns}$ on both sides of the sample. Also, the longer components of the decay of SF as well as the peaks at 3.33 and the $3.34 \mathrm{eV}$ have similar dynamics on both sides of the sample and their lifetimes are $0.61 \pm 0.05,0.69 \pm 0.05$, and $0.54 \pm 0.05 \mathrm{~ns}$, respectively. The AX also has two exponential decays with $\tau_{1}=80 \pm 5 \mathrm{ps}$ and $\tau_{2}=0.95 \pm 0.05 \mathrm{~ns}$.

The values of the FX decay constants and the shorter component of the decay $\tau_{1}$ for the DX and the SF are collected in Table 1. Intensity values of time integrated PL, averaged over a few points on the sample, are also displayed. A comparison of the values presented in Table 1 obtained for covered and uncovered parts of the sample indicates that the FX-related decay times are reduced in the presence of the Ag nanoislands. Also, the emission intensity is lower for the covered side. These effects are

Table 1 PL lifetimes (fast components) and intensities determined for free exciton (FX), bound exciton (DX), and stacking fault (SF) transitions in sample B

\begin{tabular}{|c|c|c|c|c|c|}
\hline \multirow[t]{2}{*}{ Transition } & \multirow{2}{*}{$\begin{array}{l}\text { Energy } \\
(\mathrm{eV})\end{array}$} & \multicolumn{2}{|c|}{ PL lifetime (ps) } & \multicolumn{2}{|c|}{$\begin{array}{l}\text { Intensity } \\
\left(10^{3} \text { counts }\right)\end{array}$} \\
\hline & & Plain & With Ag & Plain & With Ag \\
\hline FX & 3.375 & $35 \pm 5$ & $25 \pm 5$ & 4.7 & 4.0 \\
\hline DX & 3.36 & $77 \pm 5$ & $66 \pm 5$ & 39 & 17 \\
\hline $\mathrm{SF}$ & $3.30-3.33$ & $40 \pm 15$ & $30 \pm 10$ & 7.0 & 1.3 \\
\hline
\end{tabular}

attributed to the existence of an additional nonradiative channel related to the coupling of the emission to localized surface plasmons confined in the Ag nanoislands. A similar reduction of the decay time was previously observed [2]; however, in that case, an additional channel was of a radiative nature and led to an increase of the emission magnitude. As modeling presented in the "Theoretical model" section reveals, the damping of the emission magnitude observed in our case results from the fact that for Ag nanoparticles with diameters of $80-90 \mathrm{~nm}$ (or smaller), the absorption efficiency dominates over the scattering efficiency in the near-band gap spectral range. The Ag nanoislands influence mainly free excitons, either because they can diffuse to the surface or because spatially extended excitations are more strongly coupled to plasmons. Accordingly, the recombination rate of bound excitons is only weakly affected by Ag coating. However, the PL intensity of bound excitons is reduced, as Ag nanoislands, by constituting centers of free exciton recombination, diminish the concentration of bound excitons. Time-resolved measurements on sample A show that appropriately small Ag nanoislands have a minor effect on the PL lifetimes.

\section{Theoretical Model}

In order to describe the effects observed experimentally, we employ a model developed within the Mie scattering theory [11-13]. According to this theory, the efficiencies $Q_{\text {scat }}$, $Q_{\text {ext }}$, and $Q_{\text {abs }}$ of respectively scattering, extinction, and absorption of the light interacting with a single spherical particle of a radius $R$ are (notation according to [13]):

$$
\begin{aligned}
& Q_{\text {scat }}=\frac{\lambda^{2}}{2 \pi^{2} R^{2}} \sum_{n=1}^{\infty}(2 n+1)\left(\left|a_{n}\right|^{2}+\left|b_{n}\right|^{2}\right), \\
& Q_{\text {ext }}=\frac{\lambda^{2}}{2 \pi^{2} R^{2}} \sum_{n=1}^{\infty}(2 n+1) \operatorname{Re}\left\{\mathrm{a}_{\mathrm{n}}+\mathrm{b}_{\mathrm{n}}\right\},
\end{aligned}
$$

$Q_{\mathrm{abs}}=Q_{\mathrm{ext}}-Q_{\text {scat }}$,

with:

$$
\begin{aligned}
& a_{n}=\frac{m \psi_{n}(m x) \psi_{n}^{\prime}(x)-\psi_{n}(x) \psi_{n}^{\prime}(m x)}{m \psi_{n}(m x) \xi_{n}^{\prime}(x)-\psi_{n}(x) \psi_{n}^{\prime}(m x)}, \\
& b_{n}=\frac{\psi_{n}(m x) \psi_{n}^{\prime}(x)-m \psi_{n}(x) \psi_{n}^{\prime}(m x)}{\psi_{n}(m x) \xi_{n}^{\prime}(x)-m \xi_{n}(x) \psi_{n}^{\prime}(m x)}
\end{aligned}
$$


where

$$
\begin{aligned}
x & =k_{\text {out }} R=\frac{2 \pi n_{\text {out }} R}{\lambda}, m=\frac{k_{\text {in }}}{k_{\text {out }}}=\frac{n_{\text {in }}}{n_{\text {out }}}=\frac{\sqrt{\varepsilon_{\text {in }}}}{\sqrt{\varepsilon_{\text {out }}}}, k_{\text {in }} \\
& =\frac{\omega}{c} \sqrt{\varepsilon_{\text {in }}}, \quad k_{\text {out }}=\frac{\omega}{c} \sqrt{\varepsilon_{\text {out }}},
\end{aligned}
$$

with $\varepsilon_{\text {in }}$ and $\varepsilon_{\text {out }}$ being the dielectric functions of the particle and medium, respectively. The prime indicates differentiation with respect to a function argument. $\psi_{n}(z)$ and $\xi_{n}(z)$ are Riccati-Bessel spherical functions which can be expressed by the Bessel $J_{n+\frac{1}{2}}(z)$, Hankel $H_{n+\frac{1}{2}}^{(1)}(z)$, and Neuman $N_{n+\frac{1}{2}}(z)$ cylindrical functions of the half order.

The dielectric function of silver is assumed as in the classical Drude model taking into account the surface scattering and interband transitions:

$\varepsilon_{\mathrm{in}}=\varepsilon_{\mathrm{ib}}-\frac{\omega_{\mathrm{p}}^{2}}{\omega^{2}-i \gamma \omega}$,

$\gamma=\gamma_{\text {bulk }}+A \frac{\nu_{\mathrm{F}}}{R}$,

where $\omega_{\mathrm{p}}$ is the bulk plasmon frequency, $\gamma_{\text {bulk }}$ is a phenomenological relaxation constant of bulk silver, $v_{\mathrm{F}}$ is the Fermi velocity, $A$ is a dimensionless parameter, and $\varepsilon_{\mathrm{ib}}$ is the phenomenological parameter typically smaller than unity [14] describing a contribution of bound electrons to polarizability [15].

The Drude dielectric function parameters, $\varepsilon_{\mathrm{ib}}, \gamma_{\mathrm{bulk}}$, and $\omega_{\mathrm{p}}$, play an important role in theoretical predictions of the light/metal nanoparticle interaction. They define the position and width of the peak in extinction, scattering, and absorption efficiencies associated with the surface plasmon resonance in metal nanoparticles. Usually, the effective parameters of Drude dielectric function of pure silver, $\varepsilon_{\mathrm{ib}}=$ $3.7, \gamma_{\text {bulk }}=0.18 \mathrm{eV}$, and $\omega_{\mathrm{p}}=9.1 \mathrm{eV}$, provide a good description of the experimental data [16] and we use them in the case of calculations for sample A. However, annealing of sample $\mathrm{B}$ at a temperature of $750{ }^{\circ} \mathrm{C}$ leads presumably to interdiffusion of $\mathrm{Ag}$ and $\mathrm{Zn}$. As a result, the effective parameters might be modified, as already noted in several other cases [17-21]. In the present work, in order to describe our results for sample $\mathrm{B}$, we use $\varepsilon_{\mathrm{ib}}=8.1, \gamma_{\text {bulk }}=0.3 \mathrm{eV}$, and $\omega_{\mathrm{p}}=9.1 \mathrm{eV}$. These parameters assure a good agreement of the calculated extinction position and width with the one determined from the transmission experiment on sample B (see Fig. 5a, b).

The effective complex refractive index of the nanoparticle surrounding $n_{\text {out }}$ is defined by the "mixture rule" [22] as:

$n_{\text {out }}=f n_{\text {Air }}+(1-f) n_{\mathrm{ZnO}}$, where $f=0.66$ is a parameter resulting from a quasispherical shape of nanoislands. Furthermore, we take $n_{\mathrm{Air}}=1$ and $n_{\mathrm{ZnO}}=\sqrt{\varepsilon_{\mathrm{ZnO}}}$, where the $\mathrm{ZnO}$ dielectric function $\varepsilon_{\mathrm{ZnO}}$ is calculated following ref. [8].

In Fig. 5, the calculated spectral distributions of extinction, scattering, and absorption are shown. The calculation is made assuming that $\mathrm{Ag}$ nanoislands in the case of sample A have diameters $D$ distributed from 10 to $60 \mathrm{~nm}$ every $10 \mathrm{~nm}$, where $D=10 \mathrm{~nm}$ is taken with 0.5 weight and other diameters with equal 0.1 weight. In the case of sample B, diameter distribution from 60 to $120 \mathrm{~nm}$ every $10 \mathrm{~nm}$ with equal weight is assumed. As expected, the extinction maximum shifts to lower energies when the radius of the sphere increases: the computed energy of extinction maximum is at $\sim 3.2 \mathrm{eV}$ (sample A, Fig. 5a) and at $\sim 2.9$ or $\sim 2.4 \mathrm{eV}$

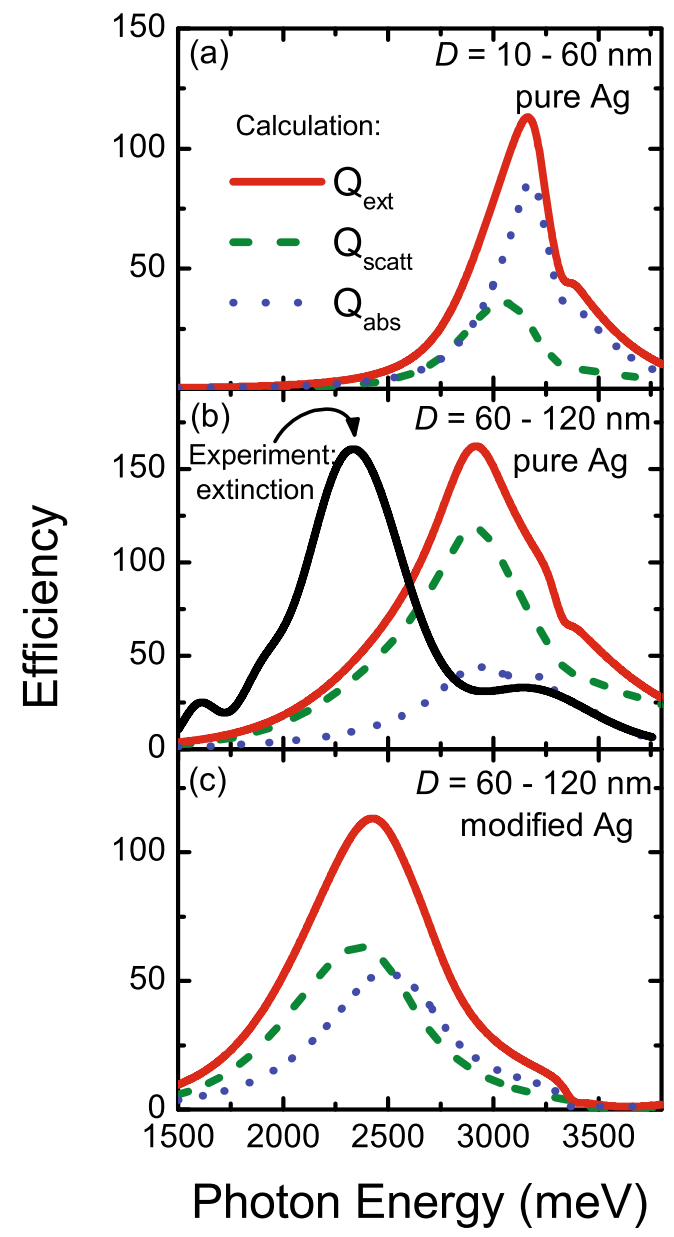

Fig. 5 Efficiency of extinction $\left(Q_{\text {ext }}\right)$, scattering $\left(Q_{\text {scat }}\right)$, and absorption $\left(Q_{\text {abs }}\right)$ calculated using Mie theory for silver nanoparticles with a diameter of $D=10-60 \mathrm{~nm}$ (a) and $D=60-120 \mathrm{~nm}(\mathbf{b}-\mathbf{c})$ deposited on $\mathrm{ZnO}$ at $300 \mathrm{~K}$. Pure silver dielectric function parameters, $\varepsilon_{\mathrm{ib}}=3.7, \gamma_{\mathrm{bulk}}=$ $0.18 \mathrm{eV}$, and $\omega_{\mathrm{p}}=9.1 \mathrm{eV}$, were assumed in (a) and (b), while modified ones, $\varepsilon_{\mathrm{ib}}=8.1, \gamma_{\mathrm{bulk}}=0.3 \mathrm{eV}$, and $\omega_{\mathrm{p}}=9.1 \mathrm{eV}$ in $(\mathbf{c})$. The extinction determined from the transmission experiment on sample B $(D=60$ $120 \mathrm{~nm}$ ) and normalized to the maximum value of the respective calculated extinction is shown in (b) 
(sample B, Fig. 5b, c) depending whether the dielectric function of pure $\mathrm{Ag}$ or the modified one is assumed, respectively. A comparison of the dashed curves in Fig. 5 affirms that an increase of the sphere radius results also in a decrease of the absorption efficiency and in an enhancement of the scattering efficiency.

In order to link these results to experimental findings, we recall that plasma oscillations excited by the incident light decay by nonradiative and radiative processes are described by absorption and scattering efficiencies, respectively. With this in mind, the computed relative intensities of absorption and scattering efficiencies explain PL and time-resolved PL experiments results. In particular, as seen in Fig. $5 \mathrm{a}$, the absorption dominates over the scattering in the whole studied spectral range in the case of sample A, leading to a decrease of the PL intensity, as observed (Fig. 3a, b).

In contrast to sample $\mathrm{A}$, in the case of sample $\mathrm{B}$, the absorption efficiency dominates over the scattering efficiency only for energies above $2.5 \mathrm{eV}$ (see Fig. 5c). As a result, a quenching of the emission is observed for energies above $\sim 2.5 \mathrm{eV}$ (see Fig. 3c, d), where excitonic transitions are visible (see Fig. 4 and Table 1). However, below $\sim 2.5 \mathrm{eV}$, a substantial emission enhancement occurs (see Fig. 3c, d).

\section{Conclusions}

In this paper, the plasmonic coupling in hybrid system $\mathrm{ZnO}$ thin film Ag nanoislands has been investigated by continuous wave and time-resolved spectroscopy. The fabrication process, involving sputtering and annealing, has served to obtain $\mathrm{Ag}$ nanoislands of dimensions insuring the presence of strong plasmonic effects. The temperature-independent enhancement or quenching of the emission is observed and explained by the numerical model developed within the Mie theory. The ratio of the absorption to scattering efficiencies is found to govern the emission enhancement or quenching. The model highlights the impact of the sample preparation on the dielectric function of Ag. The results may be useful for applications requiring a modification of the optical response of a semiconductor in the UV and visible spectral region. In particular, many groups have reported recently on the fabrication, by crystallographic or chemical phase separation, of hybrid structures consisting of magnetic metallic nanocrystals embedded in a semiconductor matrix [23]. Our results indicate dimensions of metallic nanocrystals that could lead to either enhancement or quenching of emission intensity in such heterogeneous systems.

Acknowledgments The work was supported by the European Research Council through the FunDMS Advanced Grant (\#227690) within the "Ideas" 7th Framework Programme of the EC, NCBiR project LIDER, and InTechFun (grant no. POIG.01.03.01-00-159/08). We thank P. Kossacki for critical reading of the manuscript.
Open Access This article is distributed under the terms of the Creative Commons Attribution License which permits any use, distribution, and reproduction in any medium, provided the original author(s) and the source are credited.

\section{References}

1. Okamoto K, Niki I, Shvartser A, Narukawa Y, Mukai T, Scherer A (2004) Surface-plasmon-enhanced light emitters based on InGaN quantum wells. Nature Mat 3:601. doi:10. 1038/nmat1198

2. Toropov AA, Shubina TV, Jmerik VN, Ivanov SV, Ogawa Y, Minami F (2009) Optically enhanced emission of localized excitons in $\operatorname{In}_{x} \mathrm{Ga}_{1-x} \mathrm{~N}$ films by coupling to plasmons in a gold nanoparticle. Phys Rev Lett 103:037403. doi:10.1103/ PhysRevLett.103.037403

3. Royer P, Goudonnet JP, Warmack RJ, Ferrell TL (1987) Substrate effects on surface-plasmon spectra in metal-island films. Phys Rev B 35:3753. doi:10.1103/PhysRevB.35.3753

4. Cheng P, Li D, Yuan Z, Chen P, Yang D (2008) Enhancement of $\mathrm{ZnO}$ light emission via coupling with localized surface plasmon of Ag island film. Appl Phys Lett 92:041119. doi: $10.1063 / 1.2839404$

5. Lai CW, An J, Ong HC (2005) Surface-plasmon-mediated emission from metal-capped $\mathrm{ZnO}$ thin films. Appl Phys Lett 86:251105. doi:10.1063/1.1954883

6. Wachnicki Ł, Krajewski T, Łuka G, Witkowski B, Kowalski B, Kopalko K, Domagala JZ, Guziewicz M, Godlewski M, Guziewicz E (2010) Monocrystalline zinc oxide films grown by atomic layer deposition. Thin Solid Films 518:4556. doi:10.1016/j.tsf.2009.12.030

7. Zia R, Selker MD, Catrysse PB, Brongersma ML (2004) Geometries and materials for subwavelength surface plasmon modes. J Opt Soc Am 21:2442. doi:10.1364/JOSAA.21.002442

8. Djurišić AB, Chan Y, Li EH (2003) The optical dielectric function of ZnO. Appl Phys A 76:37. doi:10.1007/s003390201290

9. Teke A, Özgür Ü, Doğan S, Gu X, Morkoç H, Nemeth B, Nause J, Everitt HO (2004) Excitonic fine structure and recombination dynamics in single-crystalline ZnO. Phys Rev B 70:195207. doi:10.1103/PhysRevB.70.195207

10. Schirra M, Schneider R, Reiser A, Prinz GM, Feneberg M, Biskupek J, Kaiser U, Krill CE, Thonke K, Sauer R (2008) Stacking fault related 3.31-eV luminescence at 130-meV acceptors in zinc oxide. Phys Rev B 77:125215. doi:10.1103/PhysRevB.77.125215

11. Mie G (1908) Ann Phys 25:377

12. Born M, Wolf E (1975) Principles of optics. Pergamon, Oxford

13. Bohren CF, Huffman DR (1983) Absorption and scattering of light by small particles. Wiley, New York

14. Derkachova A, Kolwas K (2007) Size dependence of multipolar plasmon resonance frequencies and damping rates in simple metal spherical nanoparticles. Eur Phys J-Spec Top 144:93. doi:10.1140/ epjst/e2007-00112-1

15. Bassani F, Pastori-Parravicini G (1975) Electronic states and optical transitions in solids. Pergamon, Oxford

16. Johnson PB, Christy RW, Johnson PB, Christy RW (1972) Optical constants of the noble metals. Phys Rev B 6:4370. doi:10.1103/ PhysRevB.6.4370

17. Oubre C, Nordlander P (2004) Optical properties of metallodielectric nanostructures calculated using the finite difference time domain method. J Phys Chem B 108:17740. doi:10.1021/ jp0473164

18. Ordal MA, Bell RJ, Alexander RW Jr, Long LL, Querry MR (1985) Optical properties of fourteen metals in the infrared and 
far infrared: $\mathrm{Al}, \mathrm{Co}, \mathrm{Cu}, \mathrm{Au}, \mathrm{Fe}, \mathrm{Pb}, \mathrm{Mo}, \mathrm{Ni}, \mathrm{Pd}, \mathrm{Pt}, \mathrm{Ag}, \mathrm{Ti}, \mathrm{V}$, and W. Appl Opt 24:4493. doi:10.1364/AO.24.004493

19. Blaber MG, Arnold MD, Ford MJ (2009) Search for the ideal plasmonic nanoshell: the effects of surface scattering and alternatives to gold and silver. J Phys Chem C 113:3041. doi:10.1021/ jp810808h

20. Zeman EJ, Schatz GC (1987) An accurate electromagnetic theory study of surface enhancement factors for silver, gold, copper, lithium, sodium, aluminum, gallium, indium, zinc, and cadmium. J Phys Chem 91:634. doi:10.1021/j100287a028
21. Grady N, Halas N, Nordlander P (2004) Influence of dielectric function properties on the optical response of plasmon resonant metallic nanoparticles. Chem Phys Lett 399:167. doi:10.1016/ j.cplett.2004.09.154

22. Hilger A, Tenfelde M, Kreibig U (2001) Silver nanoparticles deposited on dielectric surfaces. Appl Phys B 73:361. doi:10.1007/ s003400100712

23. Bonanni A, Dietl T (2010) A story of high-temperature ferromagnetism in semiconductors. Chem Soc Rev 39:528-539. doi:10.1039/ B905352M 